\title{
Resolución de problemas matemáticos con fracciones enfocados al contexto escolar
}

\section{Resolution of mathematical problems with fractions focused on the school context}

Fecha de recepción: 1 de Marzo de 2016.

Fecha de aprobación: 10 de Noviembre de 2016.

Artículo de Investigación
Diana Paola Avella Tuta* Fabio Abirton Salazar Pérez** José Edilberto Miguez, García***

\section{Resumen}

Este artículo da a conocer los resultados parciales de una investigación de enfoque cualitativo de tipo acción educativa, que está orientada a desarrollar una propuesta didáctica para la enseñanza de las matemáticas. La investigación se centra en el tema de la enseñanza de la resolución de problemas matemáticos con fracciones, con niños de grado sexto de la Institución Educativa Técnica Carlos Alberto Olano Valderrama del municipio de Belén (Boyacá). La propuesta consiste en orientar la enseñanza a partir de la resolución de problemas. Esta propuesta se ha realizado en tres momentos: el primero, la revisión de antecedentes y discusión teórica; el segundo, la aplicación y el análisis de resultados de la prueba diagnóstica; y el tercero, el diseño, aplicación y evaluación de la estrategia didáctica. Obteniendo que el $60 \%$ de los estudiantes se apropiaron del método satisfactoriamente, un $30 \%$ requiere de mayor acompañamiento y el $10 \%$ de los estudiantes no se apropiaron del método.

Palabras clave: resolución de problemas, contexto, estrategia didáctica, adición y sustracción con fracciones, didáctica de las matemáticas, obstáculos epistemológicos.
"Institución Educativa Técnica Carlos Alberto Olano Belén - Boyacá Colombia

dianitabella_16@yahoo.es

***Institución Educativa

Técnica Carlos Alberto Olano Valderrama, Belén Boyacá - Colombia fahisape@hotmail.com ***Institución Educativa Técnica Carlos Alberto Olano Valderrama, Belén Boyacá - Colombia jose.miguez@uptc.edu.co

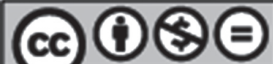




\section{Abstract}

This article discloses the partial results of a qualitative approach research of an educational action type, which is aimed at developing a didactic proposal for the teaching of mathematics. The research focuses on the subject of the teaching of solving mathematical problems with fractions, with sixth grade children of the "Carlos Alberto Olano Valderrama" Educational Institution in Belén (Boyacá). The proposal consists of focusing the teaching from the resolution of problems. This proposal has been made in three moments: the first one, the review of background and theoretical discussion; the second, the application and analysis of the results of the diagnostic test; and the third, the design, application and evaluation of the didactic strategy. Obtaining that $60 \%$ of the students appropriated the method satisfactorily, $30 \%$ required more accompaniment and $10 \%$ of the students did not appropriate the method.

Keywords: problem solving, context, didactic strategy, addition and subtraction with fractions, didactics of mathematics, epistemological obstacles. 


\section{Introducción}

El aprendizaje matemático apoyado en la resolución de problemas, es una estrategia didáctica en la que el docente presenta y resuelve con sus estudiantes, problemas del contexto social, cultural y económico de la comunidad en la que se desenvuelven las familias, siguiendo el método heurístico que propone George Polya y otros autores; el cual consiste en crear el hábito de seguir secuencias lógicas en los procedimientos que el estudiante debe aplicar, con el fin de que comprenda los conceptos matemáticos. Dicho de otra manera, la estrategia consiste en que, a partir de una situación problema del contexto, el estudiante comprenda el problema, elabore un plan, lo ejecute y haga una verificación (Polya, 2008), en el tema de adición y sustracción con cantidades fraccionarias.

De acuerdo con el tema de investigación, los autores indagaron acerca de estudios anteriores relacionados con la resolución de problemas matemáticos como estrategia didáctica para mejorar los aprendizajes de los estudiantes; los resultados arrojan alcances exitosos pero exigen compromiso, dedicación, continuidad y reflexión de carácter crítico sobre este modelo de aprendizaje por parte del docente. Del mismo modo, el presente artículo evidencia que el desarrollo de la actividad académica basada en la estrategia didáctica de resolución de problemas genera en el estudiante el hábito de leer comprensivamente y a la vez fortalece sus habilidades y competencias matemáticas, de modo que le encuentre sentido y significado a los conceptos matemáticos. Para comenzar el presente estudio, los investigadores se plantearon el siguiente cuestionamiento: ¿Cuáles son las implicaciones didácticas de la resolución de problemas con fracciones en la actividad de la enseñanza matemática en el contexto escolar, que facilitan el aprendiraje de las mismas?

De acuerdo con lo anterior, el presente artículo da a conocer los resultados parciales de una investigación en el campo de la Educación Matemática, con estudiantes de grado sexto de la Institución Educativa Técnica Carlos Alberto Olano Valderrama del municipio de Belén (Boyacá); cuyo hilo conductor es la resolución de problemas en la adición y sustracción de fracciones.

El proceso investigativo se llevó a cabo en tres momentos: el primero consistió en identificar las posibles causas por las cuales los estudiantes presentaron dificultades en la aplicación y comprensión de algoritmos para resolver problemas matemáticos. Para ello, se aplicó una prueba diagnóstica y se analizaron los resultados obtenidos en las pruebas saber de grado quinto en Matemáticas de los años 2014 y 2015. El segundo momento estuvo dedicado a la revisión de antecedentes y el estudio teórico; en el cual se abordaron los trabajos investigativos más significativos y aportantes en torno a la resolución de problemas en Matemáticas, la mayoría realizados con estudiantes de básica primaria. Esta revisión permitió identificar algunos teóricos que han desarrollado conjeturas sobre métodos para resolver problemas en Matemáticas, tales como: Polya (2008), D'Guzman (2007) y Santos (2007). El último momento consistió en planear, aplicar
El aprendizaje matemático apoyado en la resolución de problemas, es una estrategia didáctica en la que el docente presenta y resuelve con sus estudiantes, problemas del contexto social, cultural y económico de la comunidad en la que se desenvuelven las familias 
Una práctica usual en la enseñanza de las matemáticas, prioriza la mecanización por parte del estudiante de los algoritmos con los cuales pueda resolver una serie de ejercicios y evaluar una estrategia didáctica basada en el proceso heurístico de la resolución de problemas según George Polya. Para esto, se diseñaron y desarrollaron guías de clase que involucran problemas matemáticos con fracciones cercanos al entorno de los estudiantes. Se recolectó la información por medio de planillas de observación, diarios de campo, grabación de videos cortos y pruebas escritas de los estudiantes.

Su planteamiento obedece a Educación Matemática y se enmarca dentro de un enfoque epistemológico de la Didáctica de la Matemática, que integra aspectos esenciales como: algoritmo, método heurístico, contexto, enfoque de aprendizaje, teoría de resolución de problemas, relación números-esquemas.

Algunos alcances de la investigación son los siguientes:

La participación de los estudiantes durante todo el proceso de la aplicación de la estrategia didáctica, fue activa y algunos demostraron interés por alcanzar la solución al problema e incitaron a los demás a seguir los mismos pasos, quienes en un principio mostraron apatía al proceso. Son ellos quienes construyeron los conceptos matemáticos a partir de la resolución de problemas por medio del método heurístico de George Polya; en la medida en que estos se iban adentrando en la solución, se cuestionaron y se vieron obligados a analizar el camino más adecuado y coherente para resolverlo.

Se fortalecieron las habilidades comunicativas de los estudiantes, principalmente la de leer y escuchar, siguiendo el primer paso del método heurístico de George Polya, el cual consistió en leer comprensivamente el enunciado del problema matemático, y luego entre ellos discutieron y acordaron la mejor estrategia de solución del problema.

El trabajo colaborativo es esencial en el proceso, puesto que el compartir las opiniones de unos y otros contribuyó a generar conceptos nuevos e inquietudes para abordar la resolución de problemas matemáticos; además, se evidenció en la socialización de los problemas la existencia de diferentes estrategias o alternativas que los estudiantes utilizaron para llegar a una misma respuesta.

\section{Diagnóstico}

Una práctica usual en la enseñanza de las matemáticas, prioriza la mecanización por parte del estudiante de los algoritmos con los cuales pueda resolver una serie de ejercicios; esta práctica acentúa la memorización de las operaciones aritméticas que fácilmente con el tiempo el estudiante olvida y, lo que es aún más grave, es que el estudiante, aunque todavía recuerde el algoritmo no sabe en qué situación de la vida cotidiana aplicarlo (Cantoral, Montiel \& Reyes-Gasperini, 2015, p. 7). En otras palabras, el aprendizaje de la matemática no tiene, en muchas ocasiones, en cuenta al estudiante y por lo mismo él le encuentra poca utilidad; por tanto, no es de extrañar que cuando se hacen pruebas que impliquen la aplicación de algoritmos que los estudiantes conocen, pero enfocados a resolver un problema, los estudiantes se sientan extrañados y no sepan qué hacer. 
En la Institución Educativa Técnica Carlos Alberto Olano Valderrama, los estudiantes de grado sexto al resolver problemas con cantidades racionales mostraron baja comprensión del enunciado, aplicaron algoritmos no coherentes de adición y sustracción de fracciones y no verificaron el resultado obtenido con lo que se les solicitó en la pregunta del problema. Se intuyó como si no hubiesen realizado en ningún momento operaciones de adición y sustracción de fracciones, ni tampoco tienen un adecuado procedimiento en la resolución de problemas, como lo afirman Blanco y Blanco (2009).

Lo anterior se corroboró en la prueba diagnóstica aplicada, en donde se percibieron los siguientes obstáculos: la interpretación de enunciados del problema por parte delos estudiantes no es satisfactorio; es decir, el estudiante puede hacer interpretaciones según su criterio, aplica confusamente un algoritmo y hace argumentos matemáticos incompletos que no dan solución al problema.

Ante esta realidad, se pueden considerar como posibles causas generadoras de tal situación, las siguientes:

Algunos docentes de la institución utilizan un método tradicional de enseñanza sin tener en cuenta estrategias didácticas adecuadas de acuerdo con el tiempo, espacio y vivencias de los estudiantes; contribuyendo a que los estudiantes actúen pasivamente y no afloren su creatividad, en donde el docente es un transmisor de información bajo el carácter disciplinario y de estricto control.

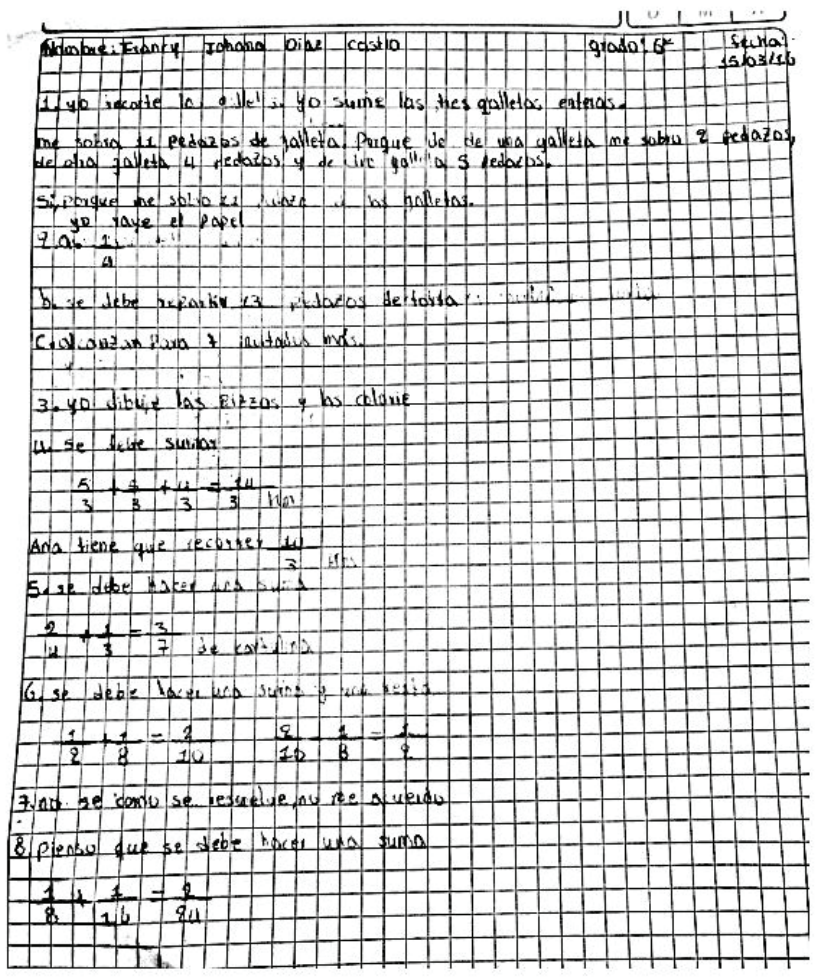

Figura 1. Prueba diagnóstica de un estudiante. Fuente: autores, junio 2016.

Los estudiantes se han creado obstáculos epistemológicos, por imaginar que las matemáticas son difíciles de aprender. Este precepto ha sido vivenciado a través de todas las generaciones, ocasionando muros que truncan el aprendizaje.

Un contexto social en el que no parece estimularse las operaciones matemáticas para resolver problemas concretos, conlleva a que el niño no relacione el aprendizaje que adquiere en la escuela con la vida cotidiana.

Hogares monoparentales o disfuncionales y con bajos niveles académicos, posiblemente influyen en los procesos de aprendizaje de los estudiantes; ya que no se promueven metas y proyectos de
En la Institución Educativa Técnica Carlos Alberto Olano Valderrama, los estudiantes de grado sexto al resolver problemas con cantidades racionales mostraron baja comprensión del enunciado, aplicaron algoritmos no coherentes de adición y sustracción de fracciones y no verificaron el resultado obtenido con lo que se les solicitó en la pregunta del problema. 
Para revalidar el bajo aprendizaje de las matemáticas de los estudiantes de grado sexto de la institución, se inició por hacer el análisis de los resultados de las pruebas saber de los años 2014 y 2015 en el área de matemáticas, del grado quinto de las sedes Central y Tuate Bajo, respectivamente. vida claros o definidos, que motiven a los aprendices a esmerarse por aprender significativamente. Esto se evidenció en el análisis a una encuesta sociodemográfica que los autores aplicaron al grupo en estudio.

Para revalidar el bajo aprendizaje de las matemáticas de los estudiantes de grado sexto de la institución, se inició por hacer el análisis de los resultados de las pruebas saber de los años 2014 y 2015 en el área de matemáticas, del grado quinto de las sedes Central y Tuate Bajo, respectivamente. Los cuales se resumen en la tabla 1.

Haciendo una comparación de los porcentajes obtenidos en la prueba por niveles de los años 2014 y 2015, se infiere que el porcentaje de estudiantes que alcanzaron un nivel avanzado en la prueba del año 2014 disminuyó en un $14 \%$, distribuyéndose este porcentaje en un $7 \%$ para el nivel satisfactorio, el cual aumentó para el año 2015; y el otro 7 \% para el nivel Mínimo, aumentando significativamente en un $11 \%$ para el año 2015.
Significativamente, el porcentaje de estudiantes que se ubicaron en el nivel insuficiente, disminuyó en un $4 \%$ del año 2014 al año 2015, lo cual es bueno para la institución. Es de resaltar que, lo que inquieta y se hace relevante en este estudio es que el porcentaje de estudiantes ubicados en el nivel mínimo aumentó, y el porcentaje de estudiantes ubicados en el nivel avanzado decayó de un año a otro. Además, se puede resaltar que los resultados de la prueba de matemáticas para el año 2015, aumentaron en un $7 \%$ en los niveles mínimo e insuficiente, pasando del $46 \%$ de los estudiantes, que se ubican en el nivel mínimo e insuficiente de desempeño en el año 2014 a un $53 \%$ en el año 2015, cabe preguntarse ¿Qué pasó?

De acuerdo con los resultados analizados en las pruebas saber de los años 2014 y 2015 del grado quinto, la institución se ubicó por encima del promedio nacional, sin embargo, esto no significa que los estudiantes estén todos en nivel avanzado o satisfactorio, sino que existe un número significativo de estudiantes

Tabla 1.

Resultados Pruebas Saber 2014 y 2015 de Matemáticas $5^{\circ}$ de la Institución Educativa Técnica Carlos Alberto Olano Valderrama

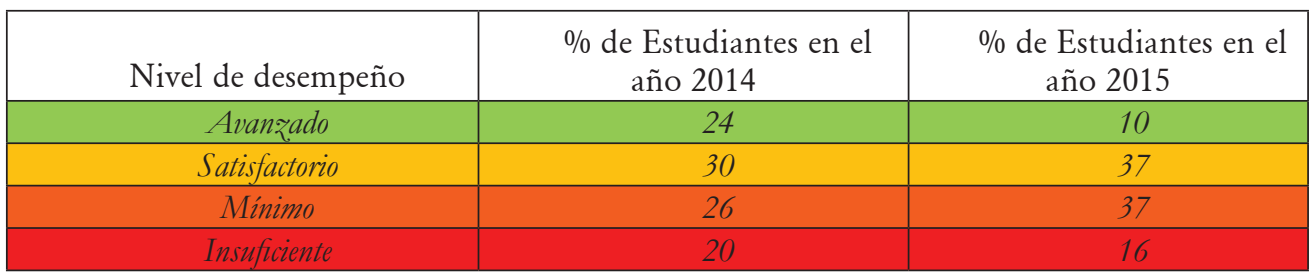

Fuente: ICFES (2015-2016)/ Índice Sintético de Calidad con interpretación de los autores. 
Tabla 2.

Comparación del nivel promedio de la institución con el promedio de Colombia en el año 2015

\begin{tabular}{|l|l|}
\hline Entidad & Valoración \\
\hline Promedio de la Institución & 328 \\
\hline Promedio de Colombia & 317 \\
\hline
\end{tabular}

Fuente: Fuente ICFES (2016)

que demuestran baja comprensión de las matemáticas, sobre todo en cuanto se refiere a la solución de problemas (Colombia Aprende, 2015, p. 27).

Por lo tanto, se hizo necesario orientar al estudiante para que aplique un proceso sistemático, que le permita comprender el enunciado del problema matemático, determinar el algoritmo u operaciones matemáticas que resuelven el problema luego de hacer análisis, para finalizar con la comprobación o verificación del plan ejecutado.

Esto se logró con problemas matemáticos cercanos al contexto del estudiante, ellos tienen la posibilidad de descubrir, comprender y aplicar los conocimientos básicos de la matemática en su vida diaria.

\section{Justificación}

Para el diseño de esta investigación, se determinó un proceso lógico que facilitó al estudiante la comprensión de los enunciados de los problemas y su resolución, con el fin de formar estudiantes con las siguientes habilidades matemáticas: interpretar, analizar, resolver, probar y comunicar la solución a problemas matemáticos; $y$, a la vez, de afianzar los conocimientos matemáticos con cantidades racionales.
Los contenidos de fracciones en el aprendizaje de los estudiantes a lo largo de la enseñanza de las matemáticas, han demostrado bastantes dificultades, como lo afirma Godino (citado en Hurtado, 2012):

Su estudio está condicionado por la progresiva comprensión de las situaciones de medición de magnitudes no discretas. Los números racionales son el primer conjunto de experiencias numéricas de los niños que no están basadas en los algoritmos de recuento como los números naturales (p. 24).

La resolución de problemas en matemáticas, implica para el docente el reto de plantear una estrategia didáctica, en la cual se implementen la modelación y manipulación de elementos tangibles, mediante los cuales el estudiante pueda hacer una representación cercana del problema, ayudándole a fijar un punto de partida y la consecución de un proceso secuencial que le permita llegar a obtener una posible solución correcta del problema, y más aún cuando este se contextualiza en el medio que rodea al estudiante.

Es así como en esta propuesta se llega a implementar una estrategia basada en el método heurístico de George operaciones aritméticas y de las
Para el diseño de esta investigación, se determinó un proceso lógico que facilitó al estudiante la comprensión de los enunciados de los problemas y su resolución, con el fin de formar estudiantes con las siguientes habilidades matemáticas: interpretar, analizar, resolver, probar y comunicar la solución a problemas matemáticos 
El diario transcurrir de la vida de las personas ya sea en el hogar, en el estudio, en el trabajo, en la recreación y muchas otras, ofrece innumerables oportunidades para vivenciar y poner en práctica el conocimiento matemático;
Polya, que contribuyó a potencializar el proceso de aprendizaje de la resolución de problemas que involucraron operaciones de adición y sustracción con fracciones; se comenzó con problemas sencillos relacionados con la vida cotidiana y los estudiantes usaron recursos didácticos a su alcance, que les permitió vivenciar los problemas planteados. Pasando a problemas con un nivel medio de dificultad, en donde los estudiantes utilizaron la modelación o representación gráfica de las diferentes situaciones problémicas, los cuales le facilitaron la comprensión del enunciado del problema y sirvió de apoyo para determinar la estrategia de solución. Se llegó, finalmente, a un nivel de complejidad en los problemas planteados, al igual que en su resolución; es decir, se logró que un $33 \%$ de los estudiantes en este nivel de dificultad comprendieran y resolvieran problemas aplicando un método abstracto.

El abordar la resolución de problemas pasando estos tres grados de dificultad, se hace del aprendizaje de los estudiantes algo verdaderamente significativo, la resolución de problemas es una estrategia didáctica que les permite a los alumnos construir el conocimiento matemático sin necesidad de memorizarlo y mecanizarlo (Hurtado, 2012, p. 25).

El aprendizaje mediante la resolución de problemas, desarrolla en los estudiantes una habilidad que permite la comprensión del sentido mismo de las operaciones o algoritmos matemáticos, aportándoles a los estudiantes herramientas para resolver diferentes tipos de ejercicios y problemas en cualquier contexto. La matemática se convirtió entonces en un saber necesario e importante que hace competentes a los estudiantes para enfrentarse a diversas situaciones del mundo y del ejercicio de la interacción ciudadanía en general.

Como señala el Ministerio de Educación Nacional (2006), refiriéndose a la formulación y resolución de problemas como uno de los cinco procesos generales fundamentales de los currículos para el aprendizaje de la matemática:

Este es un proceso presente a lo largo de todas las actividades curriculares de matemáticas y no una actividad aislada y esporádica; más aún, podría convertirse en el principal eje organizador del currículo de matemáticas, porque las situaciones problema proporcionan el contexto inmediato en donde el quehacer matemático cobra sentido, en la medida en que las situaciones que se aborden estén ligadas a experiencias cotidianas $\mathrm{y}$, por ende, sean más significativas para los alumnos (p. 52).

El diario transcurrir de la vida de las personas ya sea en el hogar, en el estudio, en el trabajo, en la recreación $\mathrm{y}$ muchas otras, ofrece innumerables oportunidades para vivenciar y poner en práctica el conocimiento matemático; también las otras ciencias ofrecen problemas y oportunidades que exigen una comprensión matemática, con lo que se puede lograr una interdisciplinariedad con otros saberes.

"Cuando el estudiante adquiere el conocimiento matemático mediante la resolución de problemas, se le permite que desarrolle la habilidad mental 
constante e insistentemente, lo cual le desarrollará la habilidad para ser estratégico a la hora de enfrentarse a cualquier tipo de problema, resolverlo y encontrar varios caminos para verificar los resultados, a la vez está en capacidad para modificar condiciones en el problema inicial y así proponer otros problemas" (MEN, 2006, p. 52).

Son varios los académicos que han estudiado y dado avances a esta teoría, al igual que los matemáticos que se han dedicado al estudio investigativo del proceso para resolver problemas, tales como George Polya, Santos Trigo, Miguel de Guzmán, quienes proponen estrategias o métodos que son muy parecidos. En esta investigación, también se tiene el propósito de determinar un método de resolución de problemas a partir de las propuestas de los matemáticos anteriormente nombrados, el cual se aplique en el desarrollo de la investigación con los niños de grado sexto de la institución.

De acuerdo con Cerda (2014), el fin de la educación matemática es contribuir en la formación de personas competentes para enfrentarse a las diversas situaciones variables de la sociedad actual; en esta dinámica, están los problemas de la vida con su diversidad de contextos. De esta manera, se contribuye en la preparación de individuos para saber atender lo que la vida del mundo moderno ofrece. Por lo tanto, la utilización de la metodología de la enseñanza basada en la resolución de problemas en las aulas escolares, es una buena herramienta (p. 24).

\section{Discusión teórica}

\section{Obstáculos en la didáctica de las matemáticas}

Para comprender las debilidades de aprendizaje que los estudiantes demostraron en la prueba diagnóstica, se consultaron teorías de algunos investigadores de didáctica de las matemáticas, quienes hablan de los obstáculos en los procesos de aprendizaje. Según D'Amore, Fandiño, Marazzani \& Sbaragli (2012), en didáctica de la matemática se distinguen tres tipos de obstáculos: ontogenéticos, didácticos y epistemológicos (p. 50).

\section{Los obstáculos ontogenéticos}

Están relacionados con las capacidades y conocimientos que tiene el sujeto de acuerdo con la edad mental; tiene relación con el desarrollo de la inteligencia, de los sentidos y de los sistemas perceptivos ( $\mathrm{D}$ 'Amore et al., 2012).

\section{Los obstáculos didácticos}

Están relacionados con las formas que cada docente utiliza para acercar a los estudiantes al concepto; el docente se vale del currículo, metodología, proyecto e interpreta de forma personal la transposición didáctica de acuerdo con sus convicciones científicas y didácticas, considerándolas efectivas; pero puede ser bueno para el aprendizaje de unos estudiantes y de otros no ( $\mathrm{D}^{\prime}$ Amore et al., 2012).
Son varios los académicos que han estudiado y dado avances a esta teoría, al igual que los matemáticos que se han dedicado al estudio investigativo del proceso para resolver problemas, tales como George Polya, Santos Trigo, Miguel de Guzmán, quienes proponen estrategias o métodos que son muy parecidos. 
Retomando la significación de obstáculo epistemológico de D 'Amore et al. (2012) y Bachelard (2004), relacionado con el aprendizaje matemático adquirido por los estudiantes

\section{Los obstáculos epistemológicos}

De acuerdo con D' Amore et al. (2012), se concibe obstáculo epistemológico "cuando en la historia de la evolución de un concepto se encuentra una nocontinuidad, un cambio radical de la concepción, entonces se asume que este concepto tiene en su interior un obstáculo, ya sea para ser concebido y aceptado" (p. 53), los estudiantes de la básica primaria, año a año, construyen obstáculos epistemológicos en el aprendizaje de algoritmos con fracciones, en el algoritmo de radicales, potenciación, entre otros; porque la enseñanza es dada por diferentes profesores, quienes transmiten los algoritmos matemáticos según la convicción de ellos; por tanto, esta situación favorece las dificultades que presentan los estudiantes en la educación básica secundaria. El filósofo francés Bachelard (2004), afirma:

Es en términos de obstáculo que se necesita poner el problema de conocimiento científico. Y no se trata de considerar los obstáculos externos como la complejidad y fugacidad de los fenómenos, ni de incriminar la debilidad de los sentidos o del espíritu humano: es en el acto mismo de conocer, íntimamente, que aparecen por una especie de necesidad funcional la extensión y la confusión. Es allí que nosotros mostraremos las causas del estancamiento incluso de regresión es allí que identificamos las causas de la inercia, que llamamos obstáculo epistemológico (p. 15).

De acuerdo con Bachelard (2004), los obstáculos epistemológicos son parte de las creencias que posee el ser humano al tener contacto con el conocimiento y su propia realidad, por tanto, los obstáculos surgen en cada estudiante y son inevitables, puesto que cada ser tiene una creencia de su realidad, que al relacionarla con el conocimiento matemático puede causar confusión.

Retomando la significación de obstáculo epistemológico de D 'Amore et al. (2012) y Bachelard (2004), relacionado con el aprendizaje matemático adquirido por los estudiantes; se entiende que los obstáculos epistemológicos dependen de la historia de la creación de los conceptos, de la evolución que dichos conceptos han adquirido en la sociedad matemática y la realidad que vivencia cada individuo al acercarse al conocimiento matemático.

\section{La resolución de problemas en matemáticas}

La resolución de problemas en el aprendizaje de las matemáticas, es una actividad presente en las clases de matemáticas, lo cual permite que el estudiante desarrolle habilidades para pensar, crear, desarrollar la curiosidad, mantener la constancia y la confianza dentro y fuera del aula de matemáticas. Una persona que sea buena solucionando problemas matemáticos, fácilmente se desenvuelve en la vida cotidiana (Giménez \& Santos, 2013, p. 49).

Para que el estudiante le encuentre sentido a lo que aprende, es importante que resuelva problemas matemáticos creados a partir de situaciones muy 
cercanas a su experiencia. La resolución de problemas matemáticos se enfrenta en el aula, siguiendo los estilos propuestos por cada uno de los siguientes teóricos: George Polya, Santos Trigo, Miguel D'Guzman, Schoenfeld, entre otros. Sin embargo, a continuación, se da a conocer la fundamentación teórica de algunos autores.

Los planteamientos que propone Polya (2008) para resolver un problema en la clase de matemáticas, están basados en un estudio juicioso sobre los métodos de solución, que se conoce como Heurístico. A continuación, se describe cada uno de los pasos que el autor plantea:

Familiarizarse con el problema: consiste en ayudarle al estudiante para que tenga un acercamiento con el enunciado del problema, e identifique y guarde en su mente el propósito del problema, despierte la intención en su memoria y retenga los aspectos más importantes (p. 51).

Trabajar para una mejor comprensión: se refiere a que el estudiante retenga el enunciado del problema en su mente, identifique las partes principales del problema a resolver; tales como los datos y la incógnita; con ellos realizar relaciones entre sí y con los detalles del problema, esto le prepara para que haga uso correcto más adelante (p. 51).

En busca de una idea útil: le permite al estudiante utilizar las partes principales del problema y sus conocimientos previos, para encontrar una idea que pueda serle útil; para ello, debe hacer relaciones entre las partes principales $y$ los detalles del problema desde diferentes puntos de vista (p. 52). Ejecución del plan: hace referencia a utilizar la idea, a partir de la comprensión del problema, la realización de operaciones aritméticas o algebraicas o geométricas que inicialmente consideró como posible solución y coloque su razonamiento formal o intuitivo en consideración de lo realizado (pp. $52-53)$.

Visión retrospectiva: consiste en volver sobre la solución, para retomarla en el problema, analizarla a detalle y considerarla desde diferentes puntos de vista, de tal forma que se convierta en obvia o se encuentre otros caminos de solución. Esta visión genera en el pensamiento del estudiante la posibilidad de adquirir y retener otros conocimientos, que le servirán para resolver otros problemas (p. 53).

El método que propone George Polya para resolver problemas, se resume en cuatro pasos básicos, los cuales son: comprensión del problema, proponer un plan, llevar a cabo el plan, y examinar la solución.

Además, para D’Guzman (2007), la presentación de un tema matemático enfocado desde la resolución de problemas, contiene los siguientes aspectos: de acuerdo con el tema, proponer una situación problema basada en historias, juegos, aplicaciones o modelos. Paso seguido, los estudiantes (de forma autónoma) manipulan y se familiarizan con la situación planteada. Enseguida, diseñan las estrategias posibles que conlleven a la solución; los estudiantes al manipular esas estrategias mediante
El método que propone George Polya para resolver problemas, se resume en cuatro pasos básicos 
Así que, Santos Trigo hace ver que el estudiante debe ser un agente activo en las clases de matemáticas mediada por la resolución de problemas, porque construye su propio conocimiento a medida que él avanza realizando una retroalimentación el ensayo diverso, elaboran herramientas existentes a través de la historia. Avanzando, eligen la o las estrategias que mejor conduce para abordar y resolver el problema. Finalmente, los estudiantes realizan una reflexión sobre el proceso ejecutado, afianzando formalmente el conocimiento en aprendizaje, y posiblemente llegue a generalizar o determinar nuevos problemas, mediante los métodos o transferencia de resultados (pp. 36-37).

La propuesta de Miguel de Guzmán se resume en cuatro pasos: acercarse al problema, determinar la o las estrategias adecuadas, seguir la estrategia(s), y revisar el proceso llevado a cabo.

Prosiguiendo nuestra teoría, el matemático y físico Santos Trigo (2007), basado en los estudios de Schoenfeld, propone como actividades importantes en la resolución de problemas en clase de matemáticas, tres aspectos esenciales. El primer aspecto es resaltar el papel de las definiciones, con las cuales se busca que el estudiante se haga partícipe en la construcción de las definiciones matemáticas, utilizando ejemplos $\mathrm{y}$ contraejemplos que forman parte importante en la construcción del conocimiento. El segundo aspecto es el método heurístico, para lo cual propone, mediante preguntas al estudiante, enfocarlo a que busque caminos para la resolución del problema, quienes deben discutir y analizar los caminos propuestos e identificar los puntos importantes considerando varios casos hasta llegar a la solución del problema; y, por último, es importante trabajar retrospectivamente, es decir analizar la solución que se ha encontrado, pues seguramente permitirá encontrar otras formas de solución. El último aspecto que propone es las construcciones, principalmente en geometría; estas permiten a los estudiantes participar activamente en la construcción del conocimiento y el docente no es el único poseedor del conocimiento, permitiendo así una corresponsabilidad de estudiante-docente para la aprehensión (pp. 111-121).

Así que, Santos Trigo hace ver que el estudiante debe ser un agente activo en las clases de matemáticas mediada por la resolución de problemas, porque construye su propio conocimiento a medida que él avanza realizando una retroalimentación; es importante que el estudiante ayude en la construcción de definiciones matemáticas, proponga métodos heurísticos y realice construcciones matemáticas que le faciliten la resolución de problemas; se trata entonces de una metodología que facilita al docente, que trabaja reflexiva y críticamente, enriquecer sus conocimientos didácticos del contenido matemático, y al estudiante encontrar el sentido y el significado de lo que está aprendiendo, un propósito que involucra tanto los contenidos a enseñar como la didáctica para hacerlo.

Por todo esto, la resolución de problemas está siendo muy estudiada e investigada por los educadores. Según Kilpatrick (citado en Pino Ceballos, 2012), "un problema es una situación o tarea en la cual una meta quiere ser lograda y una ruta directa a ella está bloqueada" (p. 12). La finalidad de la estrategia didáctica basada en la resolución de problemas, no debe ser la búsqueda 
de soluciones concretas para algunos problemas particulares, sino facilitar el desarrollo de las capacidades básicas, de los conceptos fundamentales y de las relaciones que pueda haber entre ellos.

\section{Metodología}

El enfoque de investigación es cualitativo y la metodología es investigación acción educativa. De acuerdo con Elliott (2005), la investigación acción educativa se caracteriza por analizar las situaciones escolares que vivencian los profesores, quienes pueden reflexionar sobre los problemas que se dan en la escuela o bien pueden encargarle a otro que realice la investigación. Permite al docente desarrollar la comprensión sobre su práctica pedagógica y ayudar a establecer alternativas que solventen las situaciones difíciles. Esta forma de investigación en Educación, muestra situaciones correlacionadas unas con otras, en cuanto a los problemas escolares; el método para intervenir es a partir del estudio de casos, no necesita de fórmulas teóricas para su intervención. Los actores de la investigación son los profesores, estudiantes y directivos de la institución, puesto que, entre ellos se presentan las situaciones problémicas y ellos mismos participan de las soluciones que mejoran la situación (pp. 24 - 25).

\section{Propuesta pedagógica}

Con base en la revisión teórica, los autores incorporaron la estrategia didáctica de resolución de problemas matemáticos a partir de situaciones problémicas cercanas al contexto de los estudiantes.
Pasos para resolver problemas de matemáticas:

Leer comprensivamente el problema: para que el estudiante alcance la comprensión del enunciado problémico, el profesor hace varias preguntas de tipo textual e inferencial; los cuestionamientos se hacen de forma oral, luego de que el estudiante haya leído más de dos veces el enunciado problémico, responde el estudiante que el docente elija; este estilo permite que cada estudiante esté atento al desarrollo de la clase, pues en cualquier momento puede ser abordado con una pregunta. En seguida, los estudiantes deben registrar en su cuaderno de apuntes los datos que emerge el problema y volver a escribir la pregunta a resolver.

\section{Diseñar una táctica para encarar} el problema: en esta fase se motiva al estudiante para que proponga una estrategia de solución al problema; para ello, cada estudiante realiza una estimación de cálculo mental, la cual favorece la planeación de la estrategia que resuelve la pregunta, el educando que tenga una posible solución al problema explica su propuesta ante el grupo.

Llevar a cabo el diseño táctico: es aquí donde el estudiante pone en juego todo su conocimiento y destreza matemática, para descubrir la incógnita o el dato desconocido; para ello, debe contestar la pregunta: ¿cómo lo resuelvo? En este paso, el estudiante demuestra sus habilidades, conceptos y procesos matemáticos, actitudes y su pensamiento metacognitivo, valiéndose
De acuerdo con Elliott (2005), la investigación acción educativa se caracteriza por analizar las situaciones escolares que vivencian los profesores, quienes pueden reflexionar sobre los problemas que se dan en la escuela o bien pueden encargarle a otro que realice la investigación. 


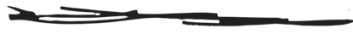

La prueba diagnóstica se aplicó con el fin de identificar el proceso que utilizan los estudiantes de grado sexto a la hora de enfrentarse a un problema de matemáticas, para compararlo con las propuestas teóricas que plantean George Polya, D’Guzmán y Santos Trigo para resolver problemas en matemáticas del conocimiento matemático (algoritmos, gráficas, modelos, métodos algebraicos, etc.). El docente desempeña un papel importante, ya que acompaña el proceso de aprendizaje del estudiante. Por último, se debe dar respuesta escrita a la pregunta del problema.

Estar seguro de la conclusión: aquí el estudiante demuestra que la solución dada es verdadera. En este paso, los estudiantes realizan la prueba del proceso utilizado en el paso anterior. Con este procedimiento, los estudiantes afianzan sus conocimientos y le encuentran sentido a la actividad realizada, además, se pueden generar nuevos problemas matemáticos.

Para la implementación de la anterior estrategia, se planearon y ejecutaron guías de clase enfocadas a la adición y sustracción de fraccionarios, que contenían textos problémicos priorizando el contexto del estudiante, bajo la salvedad que el estudiante podía adoptar cualquier camino para la solución de estos.

A medida que se desarrolló la clase, se registraron situaciones trascendentales de los procesos llevados a cabo en el aprendizaje de los estudiantes, por medio del diario de campo, la planilla de observación con la matriz FODA, se grabaron videos cortos de clase (con consentimiento informado) $y$ talleres de los estudiantes. Al analizar la información recolectada en cada uno de los anteriores instrumentos, se evidenciaron cambios tangibles en la comprensión del concepto de adición y sustracción con cantidades fraccionarias; y, a la vez, el fortalecimiento de las habilidades para resolver problemas matemáticos.

\section{Análisis de resultados}

En cuanto al análisis de resultados, se inició por evaluar la prueba diagnóstica aplicada, en seguida se retomó los resultados de las pruebas saber de los años 2014 y 2015 de matemáticas del grado quinto de la institución que anteriormente se mencionó. Paso a seguir, se consolidó la información registrada en los diarios de campo, las planillas de observación y los videos cortos de grabación de las clases, en una tabla por medio de categorías, que más adelante se describen. Posteriormente, para evaluar la estrategia didáctica, se aplicó nuevamente la prueba diagnóstica; y, al finalizar, se desarrolló una clase mediada a través del aprendizaje cooperativo, en la cual los grupos de estudiantes debían consolidar un problema matemático y determinar su solución.

La prueba diagnóstica se aplicó con el fin de identificar el proceso que utilizan los estudiantes de grado sexto a la hora de enfrentarse a un problema de matemáticas, para compararlo con las propuestas teóricas que plantean George Polya, D’Guzmán y Santos Trigo para resolver problemas en matemáticas; esto, con el fin de determinar si los estudiantes poseían una formación didáctica para resolver problemas matemáticos y/o usan un procedimiento coherente $\mathrm{y}$ completo.

Se aplicó el test con las siguientes características: un primer grupo con dos problemas, los cuales se resolvían a 

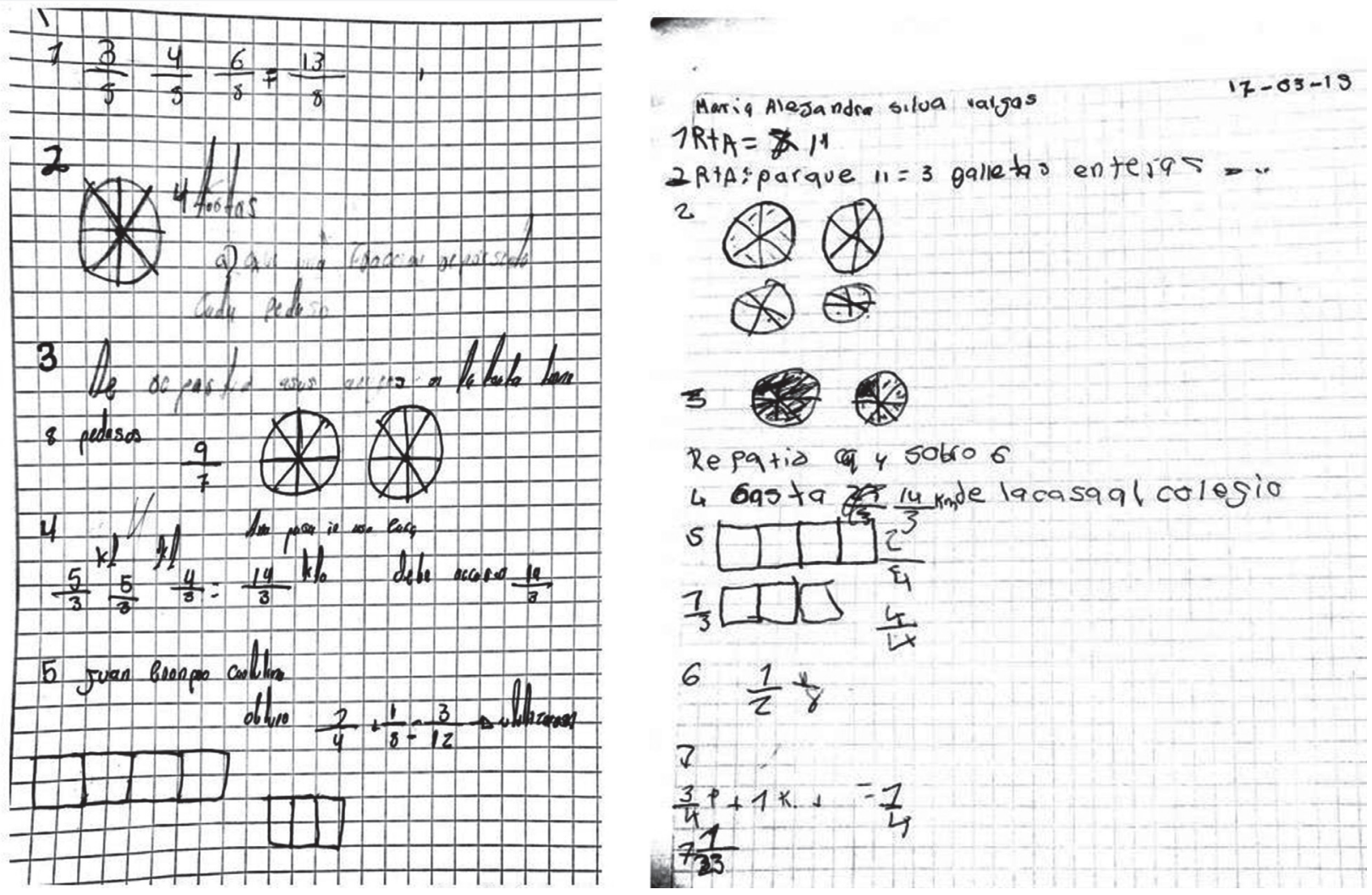

Figura 2. Prueba diagnóstica de un estudiante.

Fuente: autores, junio 2016.

partir de la manipulación de objetos que representaban la situación problémica. De este primer grupo de problemas se concluye que, los estudiantes no hacen uso correcto del material que sirve para resolver el problema, cuando lo utilizan correctamente no argumentan bien las respuestas que dan al problema. No recuerdan cómo representar simbólicamente cantidades fraccionarias, aunque algunos identifican el algoritmo a utilizar para dar respuesta a la pregunta del problema, no aplican correctamente la operación matemática. No ejecutan un adecuado procedimiento para resolver problemas, ya que, hacen una ligera lectura del enunciado problémico, no diseñan un plan para resolver, alcanzan a determinar qué operación matemática se debe aplicar, aunque no la realizan bien. Dan una respuesta a la pregunta del problema matemático, pero no hacen una reflexión de si es correcta o no. Tampoco comprueban si esa respuesta es coherente con el problema.

Ahora vamos con el segundo grupo de problemasquecontenía la representación gráfica de la situación, concluyendo que, la representación gráfica del problema le facilita al estudiante la comprensión del enunciado. Las falencias que muestran los estudiantes están en no aplicar un
De este primer grupo de problemas se concluye que, los estudiantes no hacen uso correcto del material que sirve para resolver el problema, cuando lo utilizan correctamente no argumentan bien las respuestas que dan al problema. 
El contenido de la guía ofrecía, al finalizar, una parte explicativa y demostrativa para el estudiante con un problema que ellos debían argumentar y sustentar guiándose por el modelo trabajado en el desarrollo de la clase procedimiento coherente y completo en la resolución de los problemas matemáticos, no recuerdan el proceso para aplicar adición y sustracción de fracciones homogéneas o heterogéneas, y no hacen verificación de los resultados.

Y un tercer grupo de problemas, que contaba únicamente con el enunciado del problema, encontrándose que eran más difíciles comprenderlos y darles solución, ya que solamente contaba con la escritura del enunciado. No hubo ningún estudiante que haya resuelto correctamente al menos un problema. Esto indica que los estudiantes de grado sexto de la institución no tienen claro un adecuado procedimiento que les facilite comprender y resolver problemas matemáticos, poseen falencias con el concepto de fracción y los procesos algorítmicos de adición y sustracción entre cantidades fraccionarias.

Lo encontrado en la prueba diagnóstica confirma los resultados de la prueba saber de grado quinto anteriormente mencionados.

Por tanto, los investigadores realizaron y aplicaron guías de clase que contenían objetivos claros, como: utilizar material concreto para afianzar el concepto de fracciones y comprender problemas matemáticos con dichas cantidades, enfatizar en el método que proponen los teóricos que sustentan esta investigación en la resolución de problemas, y, a la vez, promover la utilización del método a todo tipo de problemas que se enfrenta el estudiante en su vida diaria fortaleciendo así sus competencias ciudadanas.
Otro aspecto a resaltar en las guías de clase, fue la organización de los estudiantes por grupos en algunos momentos, para fortalecer el trabajo cooperativo mediante la asignación de roles, con el fin de descubrir habilidades de cada uno de los estudiantes y de esta manera identificar a aquellos que poseían facilidades de aprendizaje y liderazgo, nombrándolos como tutores.

El contenido de la guía ofrecía, al finalizar, una parte explicativa y demostrativa para el estudiante con un problema que ellos debían argumentar y sustentar guiándose por el modelo trabajado en el desarrollo de la clase, los resultados que los estudiantes obtenían en cada una de las guías fue mejorando paulatinamente el nivel de desempeño, en promedio $5 \%$ por cada una.

A continuación, se describen los hallazgos en la categorización de la información recolectada en el diario de campo, las planillas de observación y los análisis de los videos cortos de algunos momentos de la clase.

Una primera categoría que se determinó, fue el grupo general de estudiantes del grado sexto de la institución; como resultado del análisis, se subcategorizó en los siguientes aspectos: el primero, el núcleo familiar de los estudiantes; de donde resulta que, las familias son numerosas, el nivel socioeconómico está en el nivel I según SISBEN, los padres de familia y/o acudientes la mayor parte del tiempo están ocupados realizando trabajos varios. Por tanto, el apoyo emocional y económico que reciben los estudiantes es poco y en algunos hogares es visible la violencia intrafamiliar; lo 
anterior se sustenta con el análisis de la encuesta sociodemográfica aplicada. El segundo aspecto, es el nivel académico de las familias, el cual oscila entre la básica primaria y la secundaria; por consiguiente, la proyección de estudios superiores que tienen los estudiantes de la investigación es baja.

Otra subcategoría fue el comportamiento; este aspecto fue un factor relevante en cada una de las clases, ya que el grupo era numeroso y de atención dispersa; en ocasiones, el llamado a la atención llevó bastante tiempo de la clase, debido a que algunos estudiantes demostraban poco interés por las actividades presentadas por el docente.

En último lugar, la subcategoría de los horarios de clase; como se mencionó anteriormente, la presente investigación se llevó a cabo con estudiantes de grado sexto de dos sedes de la institución, la Central y Tuate Bajo; el grupo más numeroso contaba con 39 estudiantes, quienes recibían la clase de matemáticas en las últimas horas de la jornada en la sede central; esta situación afectó el éxito de la propuesta, ya que los estudiantes en esas horas se encontraban cansados mental y físicamente, por tanto no rendían lo esperado. En la sede Tuate Bajo, la docente ejerció su práctica con tres estudiantes en las primeras horas de la jornada, lo cual facilitó la receptividad y participación de los estudiantes en las clases, por tanto, la estrategia didáctica dio mejores resultados. Vale la pena aclarar que, la institución labora con jornada única, con un horario de ocho horas diarias en los niveles de educación básica secundaria.
Avanzando en nuestro análisis, la segunda categoría fue el desarrollo de la propuesta; la aplicación de los cuatro pasos para resolver problemas se subdividió en siete subprocesos, los cuales consisten en: hacer lectura comprensiva del enunciado problémico, extraer los datos, reescribir la pregunta a resolver, hacer una estimación que posiblemente facilita la respuesta a la cuestión, llevar a cabo una estrategia que proporciona solución al problema, dar respuesta formalmente a la pregunta $\mathrm{y}$, finalmente, comprobar que esa respuesta sí era la correcta.

De lo anterior, se infiere que la acogida del método fue un tanto exigente, ya que los estudiantes en un principio demostraron malestar por escribir, paso a paso, lo que debían hacer para resolver el problema. Sin embargo, poco a poco fueron accediendo y apropiándose de los subprocesos; con respecto al primero, la comprensión del problema, la mayoría de los estudiantes expresaron intuición del enunciado; puesto que, eran capaces de parafrasear el enunciado problémico e identificar los datos que son importantes para la solución del mismo y, a la vez, entendían lo que se indagaba en el problema.

Avanzando en el razonamiento, en cuanto al subproceso de llevar a cabo una estrategia para resolver el problema, en aquellos enunciados problémicos con los cuales los estudiantes contaban con el material de apoyo para vivenciar el problema, fácilmente lo manipulaban y hallaban una posible respuesta. En algunos enunciados con descripción gráfica, la estrategia buscada por los estudiantes, llevó un poco más de tiempo
En la sede Tuate Bajo, la docente ejerció su práctica con tres estudiantes en las primeras horas de la jornada, lo cual facilitó la receptividad y participación de los estudiantes en las clases, por tanto, la estrategia didáctica dio mejores resultados. 


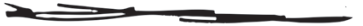

El aprendizaje de las matemáticas guiado por la resolución de problemas en el contexto, contribuye a que los estudiantes comprendan los conceptos matemáticos, y es a través de la utilización de los recursos matemáticos y dedicación; quizás por esto para algunos fue tedioso y difícil de encontrar la respuesta. Para los problemas que contenían únicamente la escritura del enunciado, los estudiantes dedicaron más tiempo y utilizaban directamente el algoritmo de sustracción y/o adición con fraccionarios; este último modelo no arrojó los resultados esperados, ya que el docente debía orientar y acompañar en todo el proceso.

Prosiguiendo con el análisis, referente al subproceso de dar la respuesta y verificarla, se evidenció que los estudiantes en pocas ocasiones escribían completamente la respuesta al problema, ya que se limitaban únicamente a dar un valor, por tanto, en su mayoría no demostraban sus argumentos.

Hay que mencionar, además, el sentir del docente en la aplicación de la estrategia; quienes vivenciaron momentos de angustia en los subprocesos que debían aplicar los estudiantes; pues, los aprendices están acostumbrados a seguir las instrucciones siempre y cuando el docente continuamente les indique y oriente. En este aspecto, se fue avanzando paulatinamente hasta lograr que un $36 \%$ del grupo logrará ser independiente en su aprendizaje. Este resultado se sustenta con el análisis de las pruebas finales presentadas por los estudiantes.

Sintetizando, para terminar el análisis con los resultados de las pruebas al finalizar la investigación, con el fin de evaluar la estrategia; se aplicó nuevamente la prueba diagnóstica y se realizó otra guía para mirar la retentiva que tenían los estudiantes.
En cuanto a la prueba diagnóstica, se evidenció que los estudiantes lograron resolver las tres clases de problemas, aplicando los subprocesos del método para resolver problemas, con algunas falencias y debilidades en el proceso; de donde se obtuvo que el $36 \%$ de ellos lograron contestar satisfactoriamente la prueba.

Se debe agregar que, el $16 \%$ de los estudiantes obtuvo una valoración cercana a la aprobación del examen; lo cual indica que ellos distinguen el proceso, pero necesitan de un mayor acompañamiento y continuidad para apropiar y afianzar el método.

Finalmente, en el trabajo por equipos, se desarrolló mediante una prueba que consistía en: organizar los enunciados de los problemas matemáticos, y poner en práctica los subprocesos para resolverlos; al terminar, un integrante del grupo socializó ante todos los compañeros. Al analizar la información recolectada en esta actividad, se llegó a que los estudiantes aplicaron la estrategia, sin embargo, cometieron errores algorítmicos y les fue difícil verificar los resultados.

\section{Conclusiones}

El aprendizaje de las matemáticas guiado por la resolución de problemas en el contexto, contribuye a que los estudiantes comprendan los conceptos matemáticos, y es a través de la utilización de los recursos matemáticos (definiciones, notaciones fundamentales, hechos básicos propios de la disciplina matemática), que el estudiante desarrolla experiencias mediante el uso de diversas 
estrategias en la resolución de problemas matemáticos relacionados con su vida cotidiana, esto genera en los estudiantes la reflexión constante y el aprendizaje significativo.

El uso de material concreto como apoyo en la solución del problema y la representación de la información del problema matemático, en algunos casos, se hace necesario. Esto le facilita la comprensión al estudiante y lo orienta a detectar la táctica que lo conduce a la solución del problema. Como cita Rosas Díaz \& Sebastián (2008) de acuerdo con las etapas del desarrollo planteadas por Piaget, el individuo a la edad de 7 a 12 años le es fácil resolver problemas mediante la modelación o manipulación de material tangible que les permita hacer una representación cercana del problema a resolver; pues, aún requieren, en cierta parte, la metodología de aprendizaje utilizada en la primaria y están en un proceso de transición para llegar a la solución de problemas con un modelo abstracto.

Es importante que el docente tenga la capacidad de reformular el problema que se aborda al inicio o utilizar un problema similar a este, para que los estudiantes descubran el camino que los conduce a la táctica de resolver el problema inicialmente planteado. Estas estrategias facilitan la comprensión y el diseño del plan de solución e implementación.

El salón de clases es un escenario propicio para desarrollar actividades del quehacer matemático, en donde los estudiantes pongan en juego valores, métodos y formas de razonamiento matemático; en la formulación y evaluación de preguntas, problemas, conjeturas, argumentos y explicaciones que se dan en el ambiente de comunidades de aprendizaje (Trigo, 2007).

El hilo conductor que se planteó en esta investigación (resolución de problemas con la adición y sustracción de fracciones), fue una notable limitante, porque fácilmente los estudiantes detectaron que, para resolver los problemas, siempre se aplicaba la misma táctica (sumar o restar fraccionarios), por tanto, no se puede afirmar un éxito de este método heurístico para toda clase de problemas matemáticos.

La propuesta pedagógica basada en los cuatro pasos para resolver problemas de George Polya, contiene implícitamente siete subprocesos que los investigadores determinaron; estos son: responder preguntas de tipo interpretativo e inferencial, extraer los datos y la pregunta del problema, realizar estimación, contestar la pregunta: ¿cómo lo resuelvo?, dar respuesta a la pregunta del problema planteado, y realizar la prueba de la solución dada.

Las prácticas pedagógicas de los docentes de matemáticas de las instituciones educativas, deben ser analizadas, reflexionadas y reestructuradas, con el fin de evaluar el quehacer pedagógico en el aula. Esto permite comprender el nivel y ritmo de aprendizaje matemático de cada uno de los estudiantes.

Al realizar un análisis sobre las propuestas metodológicas para resolver problemas de matemáticas de los autores George Polya, D' Guzmán y Santos Trigo, se infiere que los procesos son
La propuesta pedagógica basada en los cuatro pasos para resolver problemas de George Polya, contiene implícitamente siete subprocesos que los investigadores determinaron 
los mismos, ya que cada uno plantea: primero, realizar un acercamiento al problema, para ello es necesario comprender o familiarizarse con el problema por medio de situaciones problémicas cercanas al estudiante; segundo, determinar estrategias para resolver el problema, esto quiere decir utilizar todos los recursos que conoce tanto cognitivos como pragmáticos para darle solución al problema; tercero, darle solución al problema de acuerdo con el plan ejecutado; esto indica darle respuesta a la pregunta(s) del problema; y, cuarto, probar que lo realizado es correcto; con el fin de verificar o contrastar que la respuesta dada es la solución al problema.

\section{Referencias}

BACHeLARD, G. (2004). La formación del espíritu científico. En G. Bachelard, La formación del espiritu científico (pp. 15-26). México: Argos Buenos Aires.

Blanco Otano, B., \& Blanco Nieto, L. (2009). Contextos y estrategias en la resolución de problemas de primaria. Números Revista de la didáctica de las matemáticas, 71, 75-85.

Cantoral, R., Montiel, G., \& Reyes-Gasperini, D. (2015). Base de datos Universidad Pedagógica y Tecnológica De Colombia. Recuperado de http://biblio.uptc.edu. co:2092/eds/detail/detail?vid=4\&sid=761ed7ca-f12f-4c6a-bc76-accff54fded3\%4 0sessionmgr4008\&hid=111\&bdata=Jmxhbmc9ZXMmc210ZT11ZHMtbGl2ZQ $\% 3 \mathrm{~d} \% 3 \mathrm{~d} \# \mathrm{AN}=101800182 \& \mathrm{db}=$ fua

CERDA, R. S. (2014). UNIVERSIDAD AUTÓNOMA DE NUEVO LEÓN. Impacto de la resolución de problemas en el rendimiento académico en matemáticas.

Colombia Aprende. (2015). Recuperado de http://diae.mineducacion.gov.co/ siempre_diae/documentos/115087000208.pdf

D' Amore, B., Fandiño, P. M., Marazzani, I., \& Sbaragli, S. (2012). La didáctica y la dificultad en matemáticas. Bogotá: La imprenta Editores S.A.

D’Guzman, M. (2007). Enseñanza de las ciencias y las matemáticas. Revista Iberoamericana de educación, (43), 19-58.

ELLIOTT, J. (2005). La Investigación - acción en educación. Madrid: Morata.

GimÉNEZ, J., \& SANTOS, L. (2013). La actividad matemática en el aula. España: Graó.

Hurtado Orduz, M. E. (2012). Biblioteca Digital de la Universidad Nacional. Recuperado de http://www.bdigital.unal.edu.co/8573/1/01186688.2012.pdf

MEN. (2006). Estándares Básicos de Competencias. En MEN, Estándares Básicos de Competencias (p. 184). Bogotá: MEN.

Pino Ceballos, J. A. (2012). Concepciones y prácticas de los estudiantes de Pedagogía Media en Matemáticas con respecto a la Resolución de Problemas y, diseño e implementación de 
un curso para aprender a enseñar a resolver problemas. (Tesis doctoral). Universidad de Extremadura, Badajoz, España. Recuperado de http://dehesa.unex.es/ bitstream/handle/10662/568/TDUEX_2013_Pino_Ceballos.pdf?sequence=1

Polya, G. (2008). Cómo plantear y resolver problemas. México: Trillas.

Rosas Díaz, R., \& Sebastián, C. (2008). Piaget, Vygotsky y Maturana Constructivismo a tres voces. Buenos Aires, Argentina: Aique grupo editor S.A.

Santos Trigo, L. M. (2007). La resolución de Problemas Matemáticos. Fundamentos Cognitivos. México: Trillas. 